\title{
WWOX induces apoptosis and inhibits proliferation in cervical cancer and cell lines
}

\author{
JUNJIE QU ${ }^{1}$, WEN LU ${ }^{1}$, BILAN LI $^{1}$, CONG LU ${ }^{2}$ and XIAOPING WAN ${ }^{2}$ \\ ${ }^{1}$ Department of Obstetrics and Gynecology, The International Peace Maternity and Child Health Hospital \\ of China Welfare Institute affiliated with Shanghai Jiao Tong University, Shanghai 200032; \\ ${ }^{2}$ Shanghai First People's Hospital, Shanghai 200080, P.R. China
}

Received December 21, 2012; Accepted February 1, 2013

DOI: $10.3892 /$ ijmm.2013.1314

\begin{abstract}
Cervical cancer is the second most common gynecological malignancy, but the molecular events involved in its development remain unclear. The tumor-suppressor gene, WW domain-containing oxidoreductase (WWOX), has been found to be lost in various types of cancers. Few studies have been reported detailing the function of WWOX in human cervical cancer; therefore we aimed to investigate the role played by WWOX in human cervical cancer. Immunohistochemistry was used to study preinvasive and invasive primary cervical cancer. Full length cDNA was transfected into HeLa cells to overexpress WWOX, and short hairpin RNA (shRNA) was transfected into SiHa cells to deplete its expression, respectively. The cellular levels of WWOX RNA and protein were detected by real-time PCR and western immunoblotting. Proliferation rates were assessed by methyl thiazolyl tetrazolium (MTT), plate colony formation and soft agar colony assays. Cellular apoptosis was measured by flow cytometry and TdT-mediated dUTP nick-end labeling (TUNEL) assay. The activity of caspase-3 and its protein levels were determined by caspase- 3 activity assay and western blot analysis. Xenografts were established by injecting cells into nude mice. The results showed that WWOX expression was decreased in human cervical cancer and cervical cancer cell lines. Reconstitution of WWOX in HeLa cells inhibited their proliferation and induced apoptosis, while knockdown of WWOX in SiHa cells promoted proliferation and inhibited apoptosis. Xenografts in groups of mice verified the effect in vivo. These data suggest that underexpression of WWOX is
\end{abstract}

Correspondence to: Professor Xiaoping Wan, Shanghai First People's Hospital, 100 Haining Road, Shanghai 200080, P.R. China E-mail:wanxp@sjtu.edu.cn

Abbreviations: WWOX, WW domain-containing oxidoreductase; LOH, loss of heterozygosity; IHC, immunohistochemistry; MTT, methyl-thiazolyl tetrazolium; TUNEL, TdT-mediated dUTP nick end-labeling; HPV, human papilloma virus; CIN, cervical intraepithelial neoplasia; ISCC, invasive squamous cell carcinoma

Key words: WWOX, cervical cancer, apoptosis, proliferation associated with cervical cancer development. Modulation of WWOX expression may be an effective and novel method for the treatment of cervical cancer.

\section{Introduction}

Cervical cancer is the second most common malignancy of the female genital track, with an estimated 12,710 new cases and 4,290 deaths in the United States in 2011 (1). With the introduction of cervical screening by Pap smear, the testing of cervical specimens for DNA of oncogenic (high-risk) types of human papilloma virus (HPV) and the use of HPV vaccination, the incidence and mortality of cervical cancer have been dramatically reduced, but these methods are only limited to countries with highly developed health care systems (2). However $\sim 80 \%$ of cervical cancers occur in developing countries (3). In China, its incidence has increased in recent years (4), and the mortality rate has shown an increasing trend in the younger generation (5). Current approaches for treating cervical cancer have limited success; the 5-year survival rate of women with cervical cancer is estimated at $71 \%$ in the United States (1). To improve outcomes of patients with cervical cancer, it is important to investigate the molecular pathways that are critical to the development of the disease, and to identify novel targets for therapy. Cervical intraepithelial neoplasia (CIN) is the potential precursor to cervical cancer (6). It has been classified as CIN1 (mild dysplasia), CIN2 (moderate dysplasia) and CIN3 (severe dysplasia and carcinoma in situ). More recently, CIN2 and CIN3 have been combined into CIN2/3.

The tumor-suppressor gene, WW domain-containing oxidoreductase (WWOX), was first identified in 2000, and is also known as FOR and WOX1 (7). It is localized to a common fragile site referred to as FRA16D, and is located at locus 16q23.3-24.1. It encodes a protein containing two WW domains and a short-chain dehydrogenase/reductase domain (SRD) (7). The biological role of the protein is not yet well defined. The highest normal expression of this gene is detected in hormonally regulated tissues such as the testis, ovary and prostate (8), and WWOX-knockout mice show impaired gene expression of key steroidogenesis enzymes in the testis and ovary. Based on these findings, it is hypothesized that WWOX plays a role in steroid metabolism (9). 
Yet, there is still no report concerning the physiological function of WWOX in humans.

Under stress conditions, WWOX may undergo Tyr33 phosphorylation in the first WW domain and then combines to its partners such as Smad4 (10), p73 (p53 homolog) (11), c-Jun (12), CREB and NF- $\mathrm{KB}$ (13), and then relocates to the mitochondria and nuclei for inducing apoptosis. With its first detection as a tumor suppressor in breast cancer, low expression levels of the WWOX gene have been observed in many types of cancers (14-17). The low expression of WWOX is possibly due to the loss of heterozygosity or epigenetic changes, such as methylation of $\mathrm{CpG}$ islands in the promoter region. In 2010, one study found that WWOX was underexpressed in cervical cancer, but its role remains undefined (18).

In the present study, we sought to explore the role of WWOX in the pathogenesis of cervical cancer. The expression of WWOX in cervical cancer cell lines and tissues was assessed. We also investigated the effect of WWOX on cervical cancer cell proliferation, anchorage-dependent and -independent growth and apoptosis. Moreover, we confirmed this effect in nude mice in vivo. Our results indicate that WWOX is a tumor suppressor of cervical cancer.

\section{Materials and methods}

Case selection, tissue handling and pathology. Formalinfixed, paraffin-embedded surgical specimens of 75 patients presenting with normal cervix, CIN and invasive cervical cancer were examined. These tissues were retrieved from patients at the Department of Pathology at The International Peace Maternity and Child Health Hospital, affiliated with Shanghai Jiao Tong University, China, from December 2010 to November 2011. These patients ranged in age from 22 to 62 years (mean, 42 years). Eleven cases of normal cervix, 10 cases of CIN1, 22 cases of CIN2/3, 20 cases of International Federation of Gynecology and Obstetrics (FIGO) stage I invasive squamous cell carcinoma (ISCC) and 12 cases of FIGO stage II ISCCs were investigated. The diagnosis and histological classification of cervical cancer were carried out according to the criteria proposed by FIGO. The clinical characteristics of the study group are summarized in Table I. Prior written informed consent was obtained from each patient, and the study was approved by the Ethics Committee of the Medical Faculty of Shanghai Jiao Tong University.

Immunohistochemical analysis of WWOX. Sections (5- $\mu \mathrm{m})$ were placed onto poly-L-lysine-coated glass slides and air dried overnight at room temperature. Sections were dewaxed in xylene, and then rehydrated through a series of graded concentrations of ethanol. Endogenous peroxidase activity was quenched by incubating the slides in 3\% hydrogen peroxide for $10 \mathrm{~min}$.

Sections for microwave antigen retrieval pre-treatment were immersed in sodium citrate buffer ( $\mathrm{pH}$ 6.0). In brief, this was carried out by irradiating the sections in a microwave oven $(800 \mathrm{~W})$ at the highest setting for $5 \mathrm{~min}$ to permit the retrieval buffer to be boiled off. This procedure was followed by microwave irradiation at the low setting for $10 \mathrm{~min}$ in order to maintain the boiling temperature. Sections were then incubated in 5\% normal goat serum for 30 min followed by an overnight incubation with a polyclonal antibody targeted against WWOX (ab33248; Abcam, Cambridge, MA, USA) at a 1:100 dilution in phosphate-buffered saline (PBS) at $4^{\circ} \mathrm{C}$, followed by $30 \mathrm{~min}$ sequential incubations in biotinylated goat anti-rabbit secondary antibody and an $\mathrm{ABC}$ visualization detection kit (Vector Labs, Burlingame, CA, USA). Finally, sections were washed in distilled water and weakly counterstained with hematoxylin. As a negative control, the primary antibodies were omitted and replaced with preimmune serum. All slides were evaluated in a blinded manner and quantified for the percent $(\mathrm{P})$ of positively stained cells and the intensity (I) of staining. The percent of positively stained cells was arbitrarily divided into five categories (from 1 to 5): $1, \leq 10 \% ; 2,11-25 \% ; 3,26-50 \% ; 4,51-75 \%$; and 5, 76-100\%. The intensity of staining was arbitrarily divided into three categories (from 1 to 3): 1, loss of staining; 2, moderate staining; and 3, intense staining. The intensity was assessed by contrasting specimens with the positive control. From these values, the staining index was calculated according to the formula: Index of WWOX expression $=(\mathrm{P} \times \mathrm{I})$. Tumors with staining scores of 12 or 15 [intensity of staining 3 times the percent of staining score 4 or $5(>50 \%)$ ] were considered to be without loss, and all other values were grouped together as being representative of lost or reduced staining (19).

Cell culture and establishment of stable cell lines. HeLa cells were originally derived from cervical adenocarcinoma, C-33A cells were originally derived from cervical cancer, and $\mathrm{SiHa}$ cells were originally derived from cervical squamous cell carcinoma; all cell lines were obtained from the American Type Culture Collection. All cells were cultured in Dulbecco's modified Eagle's medium (DMEM)/Ham's F12 (11030; Gibco, Auckland, New Zealand) supplemented with $10 \%$ fetal bovine serum (FBS) (16000-44; Gibco, Carlsbad, CA, USA) and propagated in a fully humidified atmosphere of $5 \% \mathrm{CO}_{2} / 95 \%$ air at $37^{\circ} \mathrm{C}$. The WWOX-overexpressing plasmid was generated by GeneChem (Shanghai, China); the WWOX shRNA plasmid construct was obtained from Santa Cruz Biotechnology, Inc. (sc-44193-SH; Santa Cruz, CA, USA) and the transfection reagent was obtained from Qiagen (Shanghai, China). Transfection of cells with the WWOX-overexpressing and WWOX shRNA plasmids was carried out according to the manufacturer's instructions. To obtain a stable cell line, selection pressure was maintained by supplementing the cultures with either G418 $(400 \mu \mathrm{g} / \mathrm{ml})$ or puromycin $(1.5 \mu \mathrm{g} / \mathrm{ml})$ (both from Sigma, St. Louis, MO, USA) for a period of 2-8 weeks. Clonal populations of cells derived from the clonal ancestor, were selected by isolating single colonies of cells from each well and transferring them to a 6-well plate. The proliferation of these cells was permitted to continue in the selection medium for at least two additional passages.

Real-time RT-PCR. Total RNA was isolated from cells using Tri reagent (TR118; Molecular Research Center, Cincinnati, $\mathrm{OH}, \mathrm{USA})$. The cDNA was generated by using oligo(dT) 18 primers and a Revert Aid First Strand cDNA Synthesis Kit (K1622; Fermentas Life Science, St. Leon-Rot, Germany). A $25-\mu 1$ reaction volume was used for PCR amplification of 
single-strand cDNA using reaction conditions of 40 cycles of denaturation at $98^{\circ} \mathrm{C}$ for $10 \mathrm{sec}$, annealing at $67^{\circ} \mathrm{C}$ for $30 \mathrm{sec}$, and elongation at $72^{\circ} \mathrm{C}$ for 2 min using the PerfectShot Ex Taq kit (Loading Dye Mix, DRR05TA; Takara, Dalian, China). The primer sequences used included: WWOX forward, 5'-GAGCTGCACCGTCGCCTCTCCCCAC-3' and reverse, 5'-TCCCTGTTGCATGGACTTGGTGAAAGGC-3'; and $\beta$-actin forward, 5'-CAGCCATGTACGTTGCTATCCAGG-3' and reverse, 5'-AGGTCCAGACGCAGGATGGCATG-3'.

Duplicate reactions were performed for each sample, and the same experiment was repeated three times. The inclusion of $\beta$-actin was used as a reference gene.

Western immunoblot analysis. Cells were grown on $10-\mathrm{cm}$ dishes. After two rinses in ice-cold PBS, the cells were physically harvested and lysed in ice-cold HNTG buffer (50 mmol/1 HEPES (pH 7.5), $150 \mathrm{mmol} / 1 \mathrm{NaCl}, 10 \%$ glycerol, $1 \%$ Triton $\mathrm{X}-100,1.5 \mathrm{mmol} / 1 \mathrm{MgCl}_{2}, 1 \mathrm{mmol} / 1$ EDTA, $10 \mathrm{mmol} / 1$ sodium PPI, $100 \mu \mathrm{mol} / 1$ sodium orthovanadate, $100 \mathrm{mmol} / 1 \mathrm{NaF}, 10 \mu \mathrm{g} / \mathrm{ml}$ aprotinin, $10 \mu \mathrm{g} / \mathrm{ml}$ leupeptin, and $1 \mathrm{mmol} / \mathrm{l} \mathrm{PMSF}$ ) on ice for $30 \mathrm{~min}$. Total protein was measured using the Bio-Rad protein assay kit according to the manufacturer's instructions. Protein samples $(20 \mu \mathrm{g})$ were subsequently separated on $10 \%$ sodium dodecyl sulfate polyacrylamide electrophoresis (SDS-PAGE) gels and electrotransferred to PVDF membranes. After blocking in 5\% bovine serum albumin in Tris-buffered saline (TBS), the membranes were incubated with primary antibodies targeted against WWOX (1:1,000 dilution; Proteintech Group Inc., Chicago, IL, USA), caspase-3 (1:1,000 dilution; Abcam) and $\beta$-actin (1:2,000 dilution; Proteintech Group Inc.) at $4^{\circ} \mathrm{C}$ overnight. Membranes were washed three times in TBS containing $0.1 \%$ Triton X-100 (TBST) and incubated with a peroxidase-conjugated secondary antibody (1:1,000 dilution; Santa Cruz Biotechnology, Inc.) for $1 \mathrm{~h}$ at room temperature. The signals were developed using an ECL kit (Pierce), scanned, and analyzed using the ImageJ software program (National Institutes of Health, Bethesda, MD, USA).

Proliferation assay. Cells were seeded into 96-well plates at $2 \times 10^{5}$ cells $/ \mathrm{ml}$ and cultured in DMEM/Ham's F12 media supplemented with 10\% FBS for 1-5 days. Cell growth was documented every $24 \mathrm{~h}$ via a colorimetric assay using a 3-(4,5-dimethylthiazol-2-yl)-2,5-diphenyltetrazolium bromide (MTT) assay (Sigma). Absorbance values were collected at 490 nm using a SpectraMax 190 microplate reader (Molecular Devices, Sunnyvale, CA, USA). Control samples were treated with vehicle $(0.1 \%$ DMSO or ethanol in DMEM/Ham's F12 culture media). In each individual experiment, proliferation was determined in triplicate, and the overall experiment was repeated at least three times.

Evaluation of apoptosis. Buoyant suspension cells and attached cells were harvested and subjected to dual staining with Annexin V and propidium iodide (PI) using an Annexin V-FITC Apoptosis Detection Kit (BioVision, Palo Alto, CA, USA), according to the manufacturer's protocol. The resulting fluorescence intensities were measured by flow cytometry using a FACS flow cytometer (Becton-Dickinson, San Jose, CA, USA). Experiments were performed in tripli- cate and reproducibility was confirmed in three independent experiments.

TUNEL assay. DNA fragmentation in WWOX-transfected and non-transfected (control) HeLa and SiHa cells was assessed by TdT-mediated dUTP nick end-labeling (TUNEL) assay using the One Step TUNEL Apoptosis Kit (Beyotime, Jiangsu, China). In this assay, cell suspensions were placed onto poly-L-lysine-coated glass slides, fixed, permeabilized, and incubated with the TUNEL reaction mixture at $4^{\circ} \mathrm{C}$ overnight according to the manufacturer's protocol.

Caspase-3 activity assay. Caspase-3 activity was measured using a commercial caspase- 3 activity assay (Beyotime Institute of Biotechnology, Shanghai, China). In brief, cells were homogenized in lysis buffer. The lysate was then centrifuged at $20,000 \mathrm{x} \mathrm{g}$ for $10 \mathrm{~min}$ at $4^{\circ} \mathrm{C}$. The supernatants were incubated for $1 \mathrm{~h}$ at $37^{\circ} \mathrm{C}$ with $10 \mu \mathrm{l}$ of $2 \mathrm{mM}$ caspase- 3 substrate (Ac-DEVDpNA). Substrate cleavage was measured using a spectrofluorometer at a wavelength of $405 \mathrm{~nm}$.

Plate colony formation assay. Approximately 100 cells were seeded into each well of a 6 -well culture plate and incubated for 14 days at $37^{\circ} \mathrm{C}$ following which cells were washed twice in PBS and stained with Giemsa solution. The number of colonies containing $\geq 50$ cells was counted under a light microscope from which the plate clone formation efficiency was calculated as follows: Plate clone formation efficiency $(\%)=($ number of colonies/number of cells inoculated $) \times 100$. Each experiment was performed in triplicate.

Soft agar colony assays. Cells were seeded in $0.3 \%$ top agar in growth medium over a layer of $0.6 \%$ agar in a 6 -well plate at a density of $1 \times 10^{4}$ cells $/ \mathrm{ml}$. After 11 days of incubation, colonies of $>50$ cells were produced. Only colonies with $>50$ cells were counted and photographed with an inverted microscope. All assays were performed at least three times in triplicate.

Xenograft tumor formation assays. Two HeLa-derived cell lines (HeLa-Blank and HeLa-WWOX) and two SiHa-derived cell lines (SiHa-Blank and SiHa-WWOX-SH) were harvested and resuspended at a density of $1 \times 10^{7}$ cells $/ 200 \mu \mathrm{l}$ of sterile saline. Mice (4/group and aged 6 weeks) were injected in the subdermal space subcutaneously on the medial side of the neck with the transfected or non-transfected cells. Mice were sacrificed 30 days following the transfer of cells. Subsequently, tumors were dissected and weighed, and the tumor volume $\left(\mathrm{mm}^{3}\right)$ was measured and calculated using the formula $\left[\left(\mathrm{a}^{2} \mathrm{x} b\right) / 2\right]$; where ' $\mathrm{a}$ ' represents the smallest diameter, and ' $b$ ' represents the largest tumor diameter. The animals were housed under a laminar flow hood in an isolated room according to a protocol approved by the Animal Care and Use Committee of Fudan University (Shanghai, China).

Statistical analysis. Statistical analysis was performed using the Student's t-test or one way analysis of variance (ANOVA). All tests were completed using the Statistical Product and Service Solutions (SPSS) software program, version 16.0 (SPSS Inc., Chicago, IL, USA) or Prism (GraphPad, San 
Table I. Clinical characteristics of the study group $(\mathrm{N}=75)$.

\begin{tabular}{lccc}
\hline Clinical feature & $\begin{array}{c}\text { No. of } \\
\text { patients }\end{array}$ & $\begin{array}{c}\text { WWOX } \\
\text { lost/reduced }\end{array}$ & P-value \\
&
\end{tabular}

\begin{tabular}{|c|c|c|c|}
\hline Age (years) & & & 0.295 \\
\hline$<40$ & 28 & 18 & \\
\hline $40-49$ & 29 & 24 & \\
\hline$\geq 50$ & 18 & 13 & \\
\hline Stage & & & 0.000 \\
\hline Normal cervix & 11 & 2 & \\
\hline CIN1 & 10 & 4 & \\
\hline CIN2/3 & 22 & 19 & \\
\hline ISCC grade I & 20 & 17 & \\
\hline ISCC grade IIa & 12 & 11 & \\
\hline $\begin{array}{l}\text { Lymphatic and vascular } \\
\text { invasion in ISCC }\end{array}$ & & & 0.273 \\
\hline Negative & 12 & 11 & \\
\hline Positive & 20 & 17 & \\
\hline $\begin{array}{l}\text { Lymph node metastasis } \\
\text { in ISCC }\end{array}$ & & & 0.005 \\
\hline Absent & 24 & 20 & \\
\hline Present & 8 & 8 & \\
\hline HPV infection & & & 0.053 \\
\hline Positive & 54 & 44 & \\
\hline Negative & 21 & 11 & \\
\hline
\end{tabular}

WWOX, WW domain-containing oxidoreductase; CIN, cervical intraepithelial neoplasia; ISCC, invasive squamous cell carcinoma; HPV, human papilloma virus.

Diego, CA, USA). An $\alpha$ value of $\mathrm{P}<0.05$ was considered to indicate a statistically significant result.

\section{Results}

WWOX is underexpressed in cervical cancer tissues and cell lines. Immunohistochemical staining showed that the WWOX protein was predominantly localized to the neuclei of cervical epithelial cells, particularly in the basal layer of the epithelium. There was strong staining in the normal cervix and CIN1, whereas lost or reduced immunostaining was noted in CIN2/3 and ISCCs (Fig. 1A).

To account for both staining intensity and the uniformity of staining, a composite histoscore (percentage of the epithelium staining $\mathrm{x}$ staining intensity) was calculated. Nuclear WWOX expression (WWOX composite histoscore) was significantly lost or reduced as the cervical cancer progressed $(\mathrm{P}<0.05)$ (Table I). In addition, reduced WWOX expression was significantly associated with lymph node metastasis (Table I). These results indicate a strong association between decreased expression of WWOX and the development of cervical cancer.

To investigate whether WWOX is also underexpressed in cervical cancer cell lines, we assessed the expression of WWOX in three cervical cancer cell lines (HeLa, SiHa and
C-33A) by real-time PCR and western immunoblot analysis. Normal cervical epithelial tissues were obtained from patients who underwent hysterectomy due to myoma or adenomyosis. The mRNA (Fig. 1B) and protein (Fig. 1C) expression of WWOX were decreased in all three cell lines and this was particularly prominent in both HeLa and C-33A cells.

WWOX inhibits cell growth. To further explore the role of WWOX in cell proliferation and apoptosis, we sought to overexpress WWOX by stable transfection of the WWOX plasmid construct into the HeLa cell line which expresses a low level of the WWOX protein. As shown in Fig. 2A, the protein expression level of WWOX was increased significantly. The effect of WWOX overexpression on cell proliferation was detected by MTT assay. The MTT assays revealed that stable overexpression of WWOX significantly decreased HeLa cell growth (Fig. 2C).

To further investigate the effect of WWOX on cell proliferation, we performed shRNA-mediated stable knockdown of WWOX in SiHa cells in which WWOX protein expression is relatively high (Fig. 2B). As shown in Fig. 2D, knockdown of WWOX in SiHa cells promoted their ability to proliferate. Thus, WWOX is capable of inhibiting cell growth.

We next investigated whether WWOX is required in anchorage-dependent and -independent growth, a hallmark of oncogenic transformation (20). In HeLa cells transfected with the WWOX vector, the plate colony formation (Fig. 3A) and soft agar assays (Fig. 3C) showed that there was a significant decrease in the number and size of colonies when compared to the cells transfected with a control vector or parental wildtype cells. In contrast, stable knockdown of WWOX promoted plate colony formation (Fig. 3B) and proliferation in soft agar assays (Fig. 3D) in SiHa cells. These findings indicate that WWOX inhibits cervical cancer cell proliferation.

WWOX promotes apoptosis. The apoptotic ratio was determined by flow cytometry (Fig. 4A) and TUNEL assay (Fig. 4B). HeLa-WWOX cells displayed enhanced apoptosis when compared to their non-transfected counterparts (HeLa and HeLa-Blank control groups stably transfected with the p-EGFP-N1 control plasmid). In contrast, in SiHa cells transfected with the WWOX shRNA, the apoptotic ratio was significantly reduced (Fig. 4B).

To further elucidate the mechanism by which WWOX functions, we tested the activation and protein expression of caspase-3, a crucial mediator of programmed cell death (21). We found that overexpression of WWOX in HeLa cells markedly induced caspase-3 activation (Fig. 4C) and protein expression (Fig. 4D). Conversely, knockdown of WWOX by shRNA in SiHa cells decresed the caspase-3 activity (Fig. 4E) and protein expression (Fig. 4F). Our results indicate that WWOX plays an essential role in promoting apoptosis in cervical cancer cells.

WWOX inhibits xenograft formation in vivo. To further confirm the role of WWOX in cell proliferation, a human tumor xenograft model was established in a nude mouse model (Fig. 5). At the conclusion of this assay, it was found that the WWOX-overexpressing xenografts displayed reduced average tumor size and weight as compared with the HeLa 
A

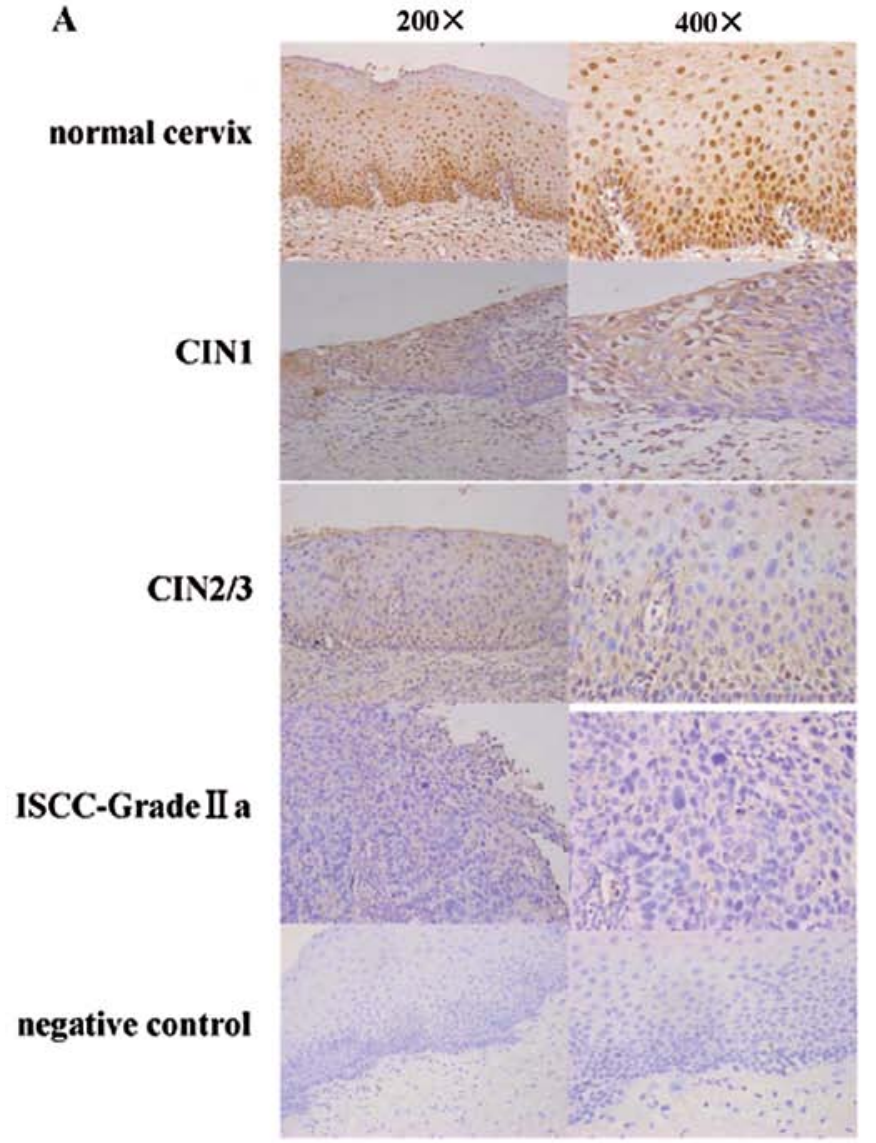

B

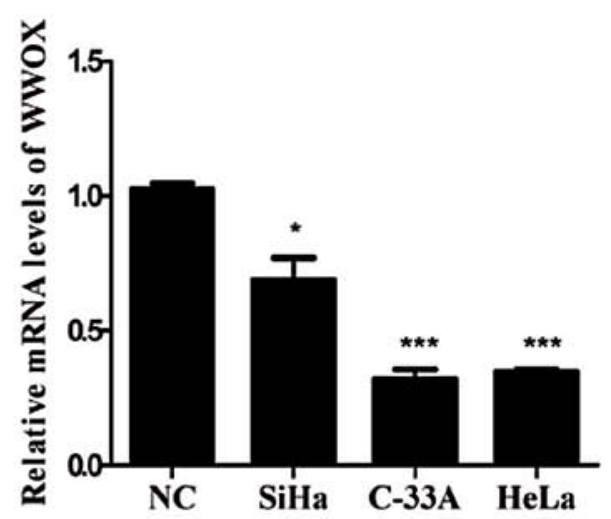

C

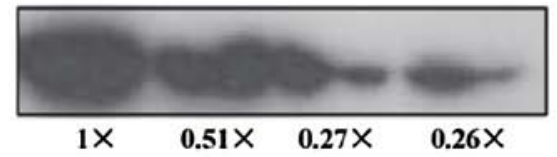

wwox

$\beta$-actin

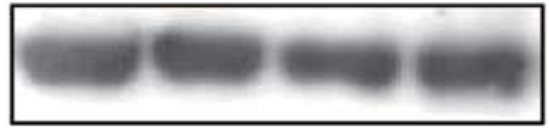

NC SiHa C-33A HeLa
B

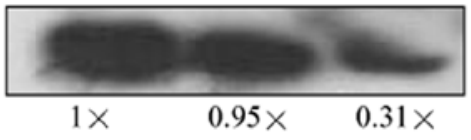

wwox

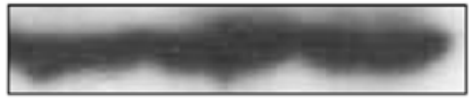

$\beta$-actin

HeLa HeLa-Blank HeLa-Wwox

SiHa SiHa-Blank SiHa-WwOX-SH

C

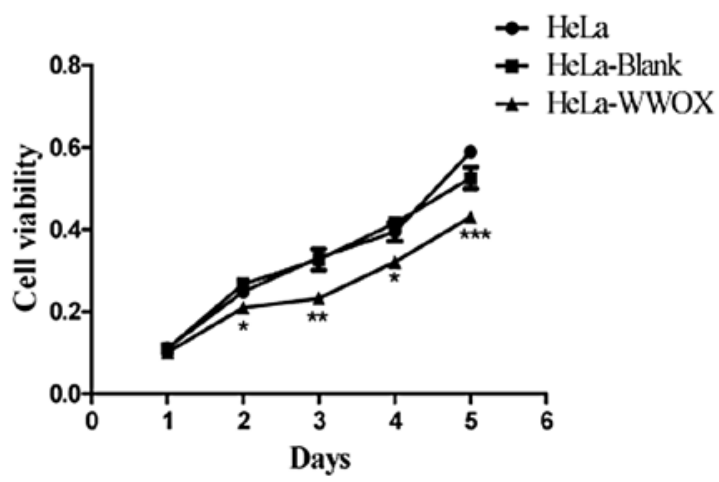

D

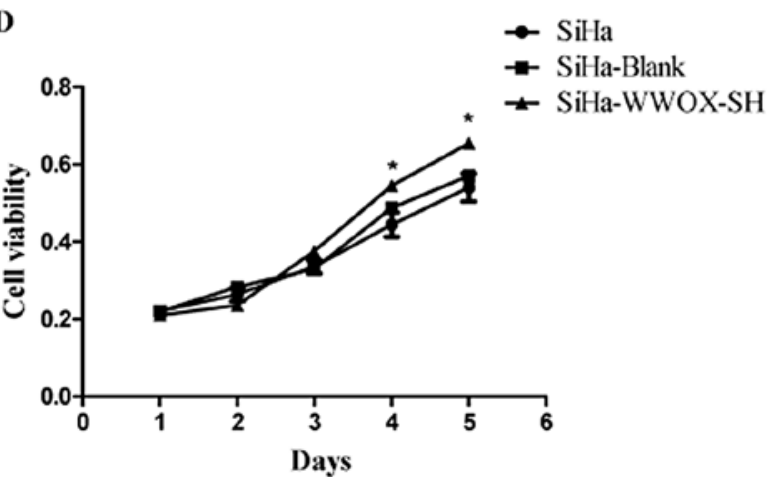

Figure 2. WWOX inhibits cervical cancer cell proliferation. (A) Protein expression of WWOX was measured in HeLa cells with stable overexpression of WWOX by western blot analysis. (B) shRNA effectiveness was confirmed by significantly decreased WWOX protein expression in SiHa cells. (C) MTT assay was applied for determining cellular proliferation in HeLa cells stably transfected with WWOX expression vector (HeLa-WWOX) or control vector (HeLa-Blank) or in non-transfected cells (HeLa); ${ }^{*} \mathrm{P}<0.05,{ }^{* * * *} \mathrm{P}<0.001$. (D) MTT assay was applied for determining cellular proliferation in SiHa cells stably transfected with the shRNA vector (SiHa-WWOX-SH) or control vector (SiHa-Blank) or in non-transfected cells (SiHa); ${ }^{*} \mathrm{P}<0.05,{ }^{* * * *} \mathrm{P}<0.001$. 
A
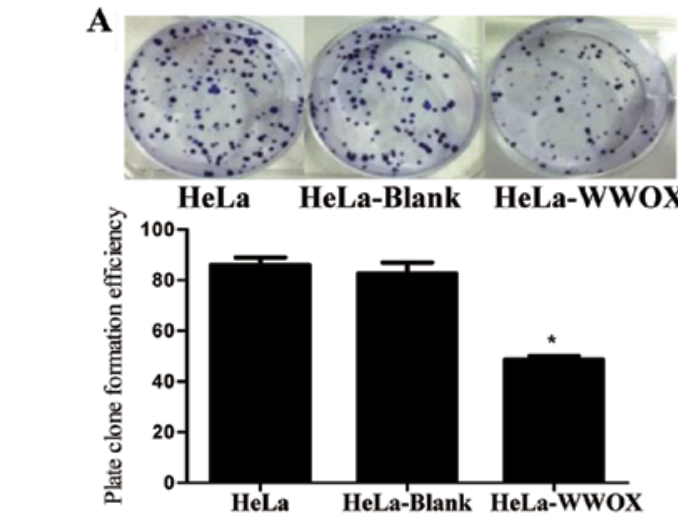

3 days
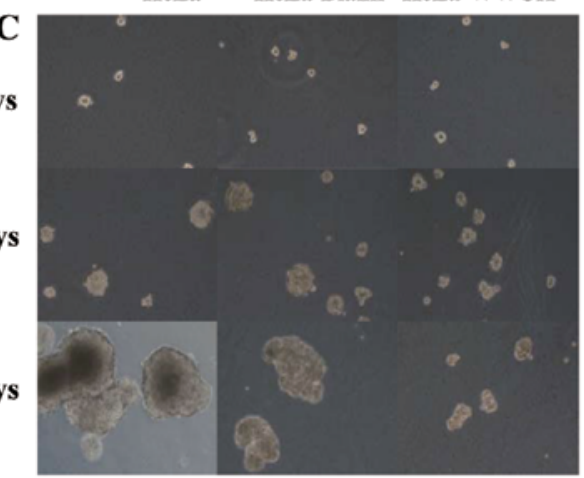

HeLa HeLa-Blank HeLa-WWOX

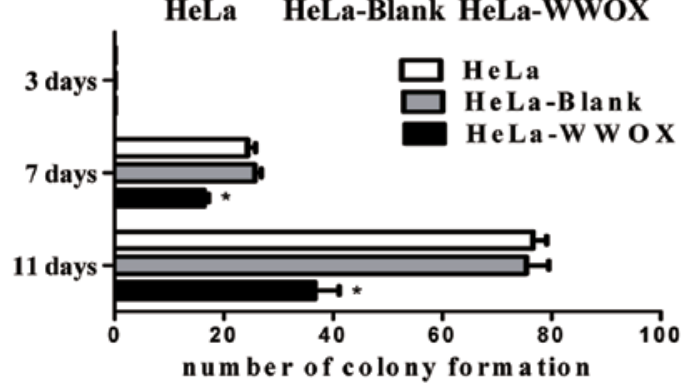

B

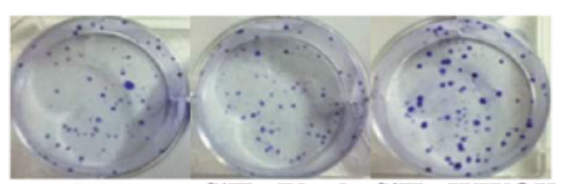

SiHa SiHa-Blank SiHa-WWOX-SH
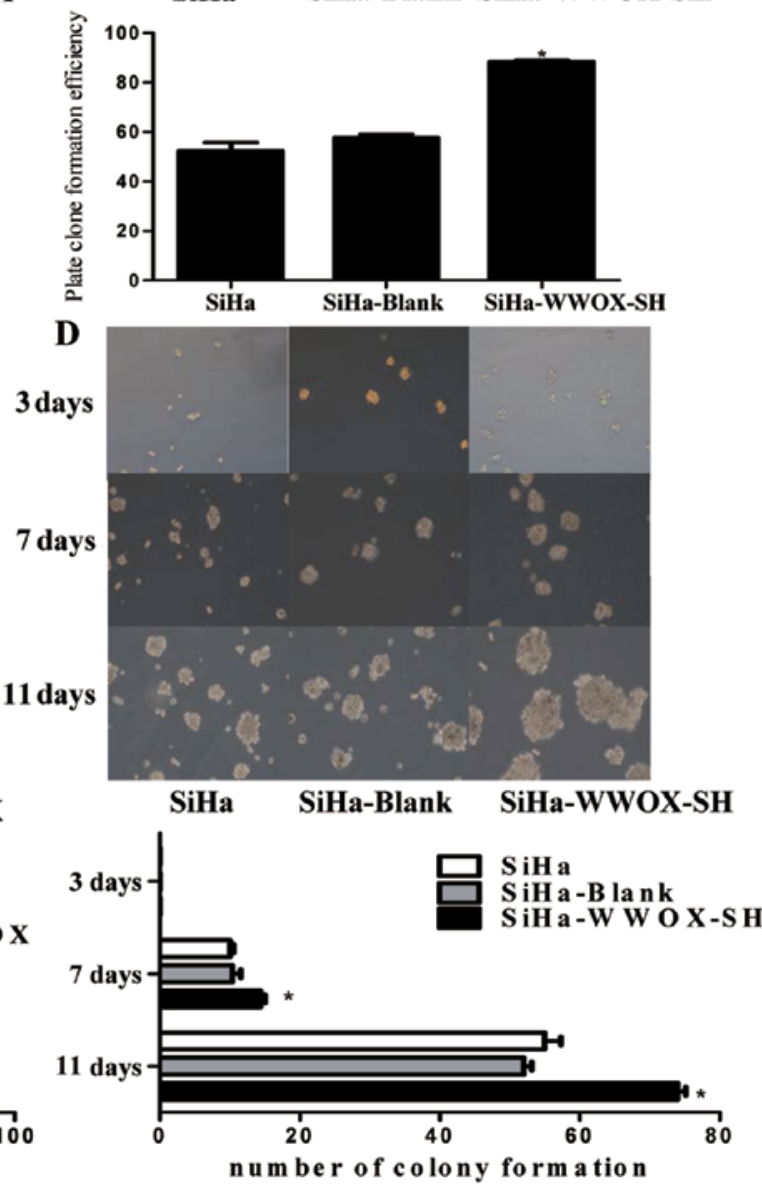

Figure 3. WWOX inhibits cervical cancer cell growth both in an anchorage-dependent and -independent manner. (A) Stable cell lines expressing WWOX were assayed for anchorage-dependent growth in a plate colony formation assay. Overexpression of WWOX led to a significant decrease in anchoragedependent colony-forming ability of HeLa cells. HeLa cells and HeLa-Blank that were stably transfected with the empty vector were used as controls. Colony formation ( $\geq 50$ cells) was assessed using a colony counter. (B) Knockdown of WWOX led to a significant increase in anchorage-dependent colony-forming ability of SiHa cells. (C) Soft agar colony assays showed that overexpression of WWOX led to a significant decrease in anchorage-independent colonyforming ability of HeLa cells. (D) Knockdown of WWOX led to a significant increase in anchorage-independent colony-forming ability of SiHa cells. (Upper panels) Representative images were captured with an inverted phase contrast microscope (magnification, x200). (Bottom panels) Columns, mean number of colonies from three independent experiments, each in triplicate; bars, $\mathrm{SD} ;{ }^{*} \mathrm{P}<0.05$.

control group (Fig. 5A). In contrast, the average size and weight of the tumors were significantly increased after mice were transfected with WWOX-knockdown cells as compared with the SiHa control group (Fig. 5B). Furthermore, the difference in tumor size between the HeLa and SiHa control groups may partially suggest the importance of WWOX in tumor formation. Collectively, these data indicate that genetic disruption of WWOX expression increases xenograft proliferation, while overexpression of WWOX inhibits xenograft tumor formation. These observations indicate a critical role for WWOX in tumor formation in vivo.

\section{Discussion}

WWOX is a tumor suppressor in several human tumor types (14-17). However, its role in cervical cancer is still unknown. In the present study, WWOX protein expression was significantly reduced as the cervical cancer progressed. And the decreased WWOX expression is related to lymph node metastasis. To further explore the possible role of WWOX in cervical cancer, we tested three cell lines derived from cervical cancer. These cell lines included HeLa (adenocarcinoma, $\mathrm{HPV}^{+}$), $\mathrm{SiHa}$ (squamous carcinoma, $\mathrm{HPV}^{+}$) and $\mathrm{C}-33 \mathrm{~A}\left(\mathrm{HPV}^{-}\right)$. Due to the lack of a normal cervical epithelium cell line, we used normal cervical tissue lysate as a normal control (16). As shown in Fig. 1C, the protein expression in cervical cancer cell lines was significantly reduced, particularly in the HeLa and C-33A cell lines. This observation was inconsistent with a previously published study (18), in which it was demonstrated that the protein expression of WWOX was absent or underexpressed in all cell lines chosen with the notable exception of Caski cells. This may be due to the 
A
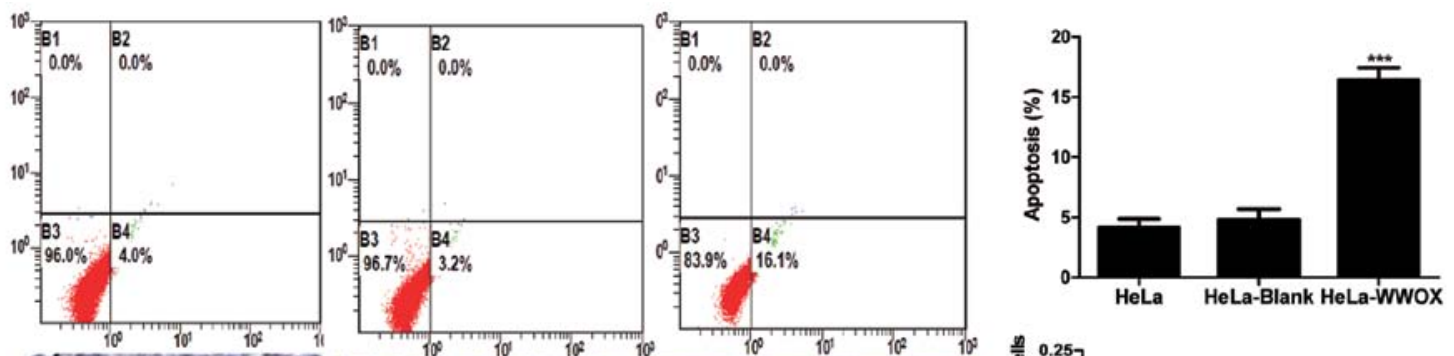

B
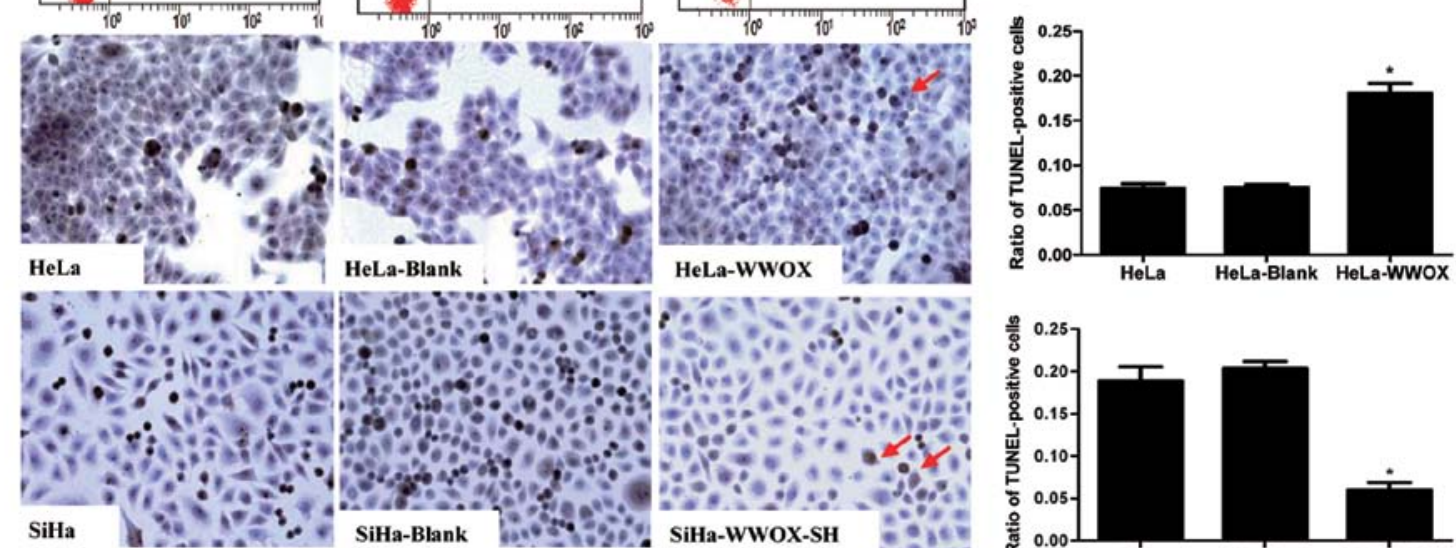

C
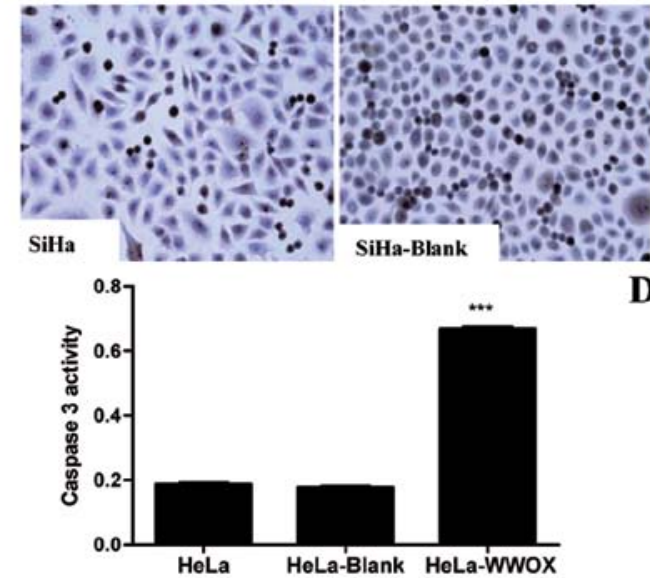

$\mathbf{E}$

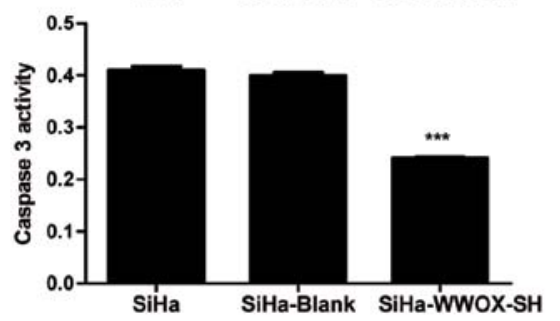

D

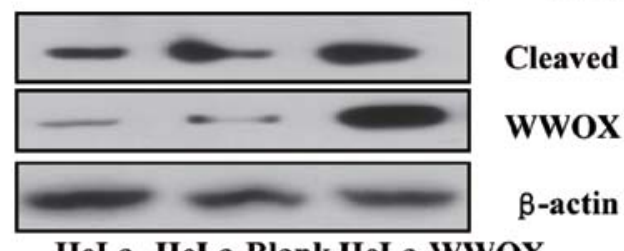

HeLa HeLa-Blank HeLa-Wwox

F

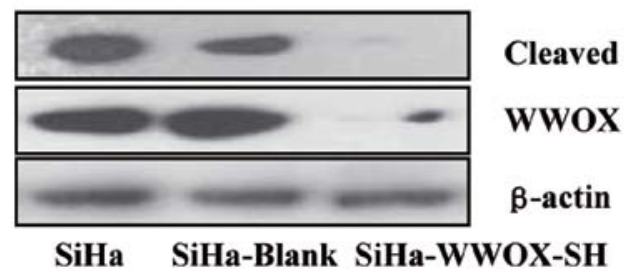

Figure 4. WWOX induces cell apoptosis. (A) Cell apoptosis was measured by flow cytometry using Annexin V-PE or PI/7-AAD double staining. HeLa and HeLa-Blank cells stably transfected with control vector were used as controls. HeLa-WWOX cells overexpressing WWOX showed obvious elevated apoptosis when compared with the controls. (B) Cell apoptosis was measured by TUNEL staining. The arrows indicate TUNEL-positive cells. (Upper panels) Overexpression of WWOX increased the TUNEL staining, (bottom panels) while knockdown of endogenous WWOX decreased the number of TUNEL-positive cells. (C and D) Caspase-3 activity and protein expression were measured by caspase-3 activity assay and western blotting in HeLa, HeLaBlank and HeLa-WWOX cells. (E and F) Caspase-3 activity and protein expression were measured in SiHa, SiHa-Blank and SiHa-WWOX-SH cells. The data are presented as means $\pm \mathrm{SD} ;{ }^{*} \mathrm{P}<0.05,{ }^{* * *} \mathrm{P}<0.001$.

different antibody and control used. In the future, the primary cultured normal cervical epithelial cells will be included to confirm this effect.

The immunohistochemical assay suggests that WWOX expression is decreased with cervical cancer progression. We believe that underexpression of the WWOX protein promotes tumor progression in cervical cancer cells. Therefore, we established models of WWOX overexpression by stable transfection of HeLa cells with the WWOX plasmid (Fig. 2A). In addition, cells with knockdown expression of WWOX were established by stable transfection of WWOX shRNA into SiHa cells (Fig. 2B). We noted that by altering the expression of WWOX, cells showed highly variable proliferative characteristics (Figs. 2C and D and 3).
Apoptosis plays a central role in tumor development, and a lack or failure of apoptosis leads to the development of many types of tumors, including cervical cancer $(22,23)$. This suggests that induction of apoptosis in tumor cells may be an effective approach for delaying tumor progression. In this study, we found that overexpression of WWOX induced apoptosis in the cervical cancer cell line HeLa (Fig. 4A and B).

We further investigated the possible pathway through which WWOX induces apoptosis. Previous research has established that caspase-3 is a principle effector caspase of apoptosis and that the intrinsic pathway of apoptosis is initiated by mitochondrial damage which in turn promotes activation of caspase-3 (24). In the cytosol or on the surface of the mitochon- 
$\mathbf{A}$

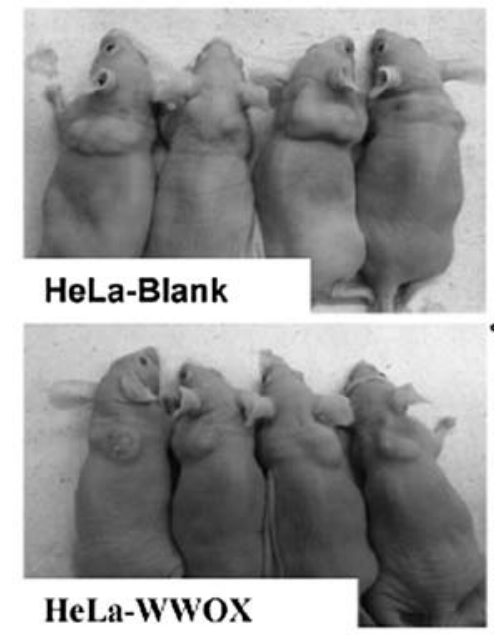

B
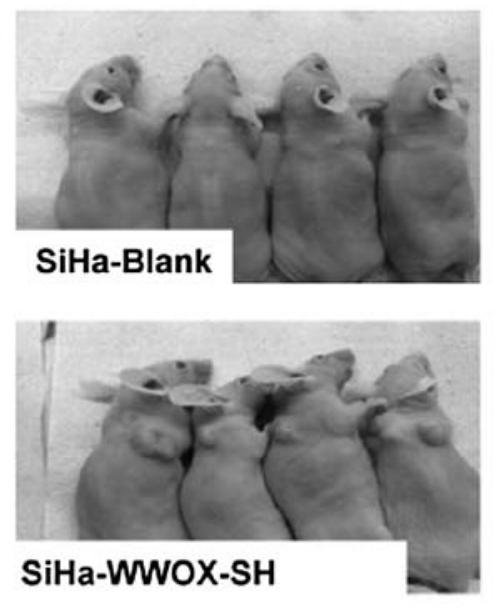
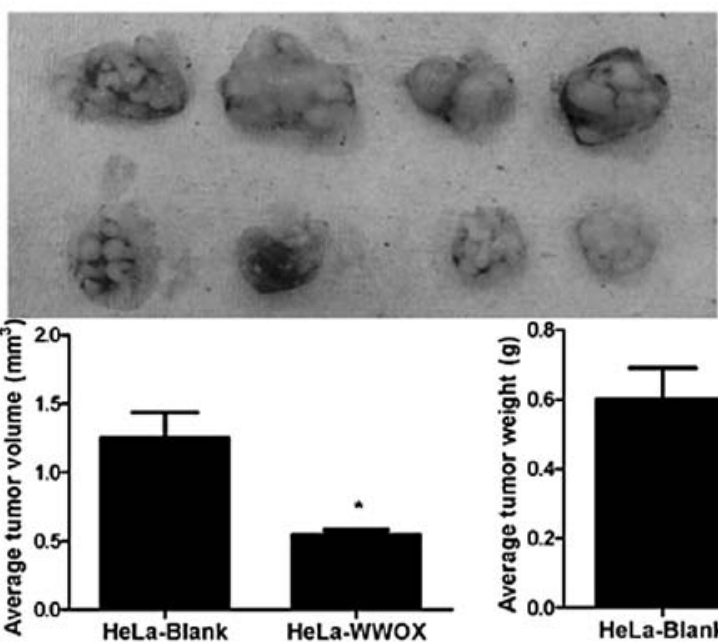

HeLa-Blank
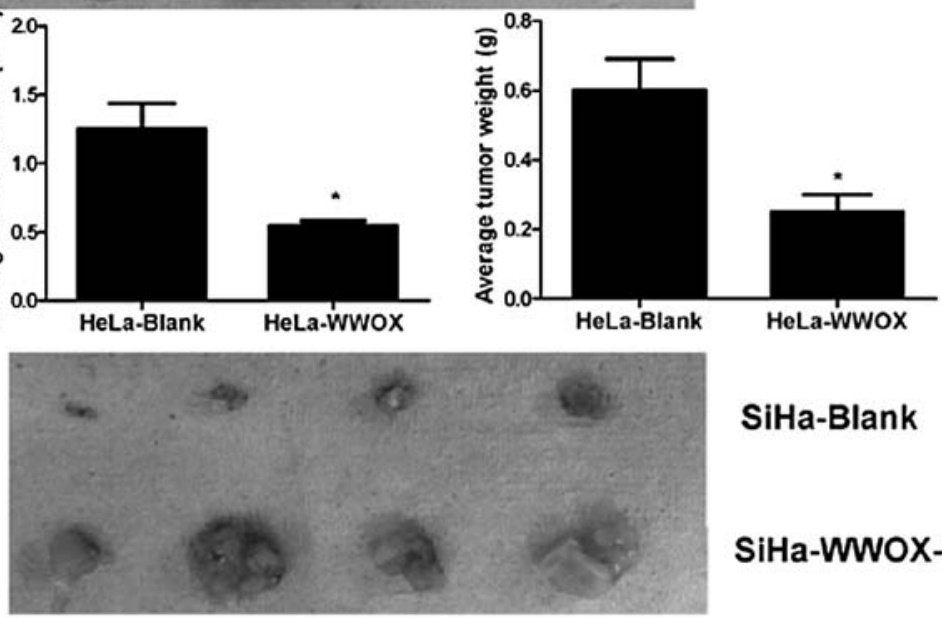

SiHa-Blank

SiHa-WWOX-SH
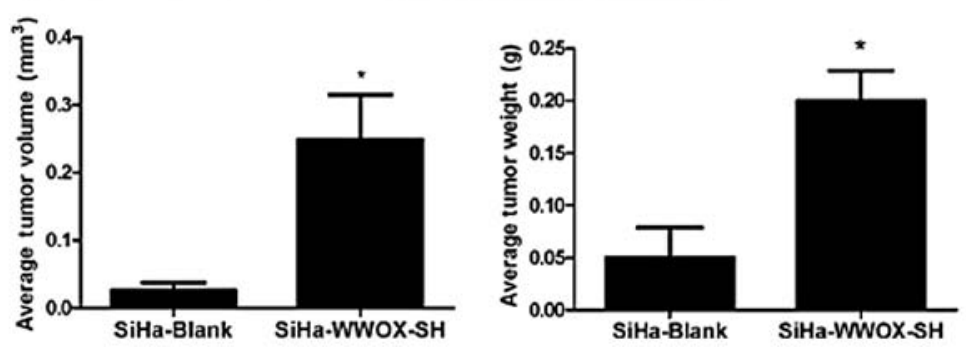

Figure 5. WWOX inhibits tumor formation in vivo. (A) Overexpression of WWOX suppressed tumor formation in nude mice. (B) Knockdown of WWOX promoted tumor formation in nude mice. The columns show the statistical results of tumor size (left) and weight (right). The data are presented as means \pm SD. ${ }^{*} \mathrm{P}<0.05$.

dria, cytochrome $c$ is bound to the protein Apaf-1 (apoptotic protease activating factor), which activates the initiating caspase, which then activates caspase-3 $(25,26)$. In western immunoblot analyses and caspase-3 activity assays, both HeLa and $\mathrm{SiHa}$ cells underwent apoptosis by a WWOX-dependent caspase-3-mediated signaling pathway. This observation was consistent with studies conducted in hepatoma and pulmonary cell lines $(27,28)$ and by contrast, was inconsistent with research carried out in a glioblastoma cell line model (29). We suspect that such differences were partly due to the different cells used in the respective research models.

Next, the notion that disruption of the normal expression of WWOX may promote tumor formation, and that, by contrast, overexpression of WWOX may inhibit tumor formation was demonstrated in an in vivo model using nude mice (Fig. 5). Overexpression of WWOX caused tumors to display smaller sizes and weight, while silencing of WWOX expression promoted the reverse effect by enhancing tumor size and weight. Collectively, these observations imply an important role for WWOX as a determinant of tumor proliferation.

The WWOX gene is located on chromosome 16q, and the common mutated form of this gene is due to the loss of heterozygosity $(\mathrm{LOH})$, which has been confirmed by several studies using various cancers including breast cancer (30), esophageal squamous cell carcinoma (14), gastric cancer (15), pancreatic cancer (16) and lung cancer (17). However, to our knowledge, there is no evidence showing WWOX LOH in cervical cancer. It was previously shown that there is loss of $16 q$ in $20 \%$ of cervical squamous cell carcinomas (31) and $38 \%$ of cervical adenocarcinomas (32). Therefore, we speculated that $\mathrm{LOH}$ in $16 \mathrm{q}$ in the context of cervical cancer may be due, at least in part, to the loss in functional expression of WWOX. This requires further direct study.

The integration of HPV into the host genome is regarded as a key step in the progression from cervical intraepithelial CIN to invasive carcinoma $(33,34)$. However, there is still a proportion of cervical cancers that show no evidence of infection by HPV (35). In contrast with these findings, we found that WWOX was reduced in C-33A cells (which is a HPV-negative cell line). This suggests that WWOX may be another important factor in tumor formation, irrespective of HPV infection. However, the possibility that HPV integration may produce a zone of fragility prone to breakage, loss and gene rearrangements (including WWOX), cannot be excluded. Thus, it is necessary to further explore the relationship between these factors. 


\section{Acknowledgements}

We thank Dr Huijuan Zhang and Mrs Yuan Liu for their help in sample collection. This study was supported by grants from the National Natural Science Funds of China (nos. 81072139, 81172476 and 81272885) and Research Fund for the Doctoral Program of Higher Education of China (20120073110090).

\section{References}

1. Siegel R, Ward E, Brawley O and Jemal A: Cancer statistics, 2011: the impact of eliminating socioeconomic and racial disparities on premature cancer deaths. CA Cancer J Clin 61: 212-236, 2011.

2. Mayrand MH, Duarte-Franco E, Rodrigues I, et al: Human papillomavirus DNA versus Papanicolaou screening tests for cervical cancer. N Engl J Med 357: 1579-1588, 2007.

3. Kent A: HPV vaccination and testing. Rev Obstet Gynecol 3: 33-34, 2010.

4. Wang Y, Chen J, Zhang W, Hong W and Yu F: Study of the prevalence of human Papillomavirus infection in Chinese women with cervical cancer. Afr J Microbiol Res 6: 1048-1053, 2012.

5. Aleyamma M and Preethi SG: Trends in incidence and mortality rates of squamous cell carcinoma and adenocarcinoma of cervix - worldwide. Asian Pac J Cancer Prev 10: 645-650, 2009.

6. Misson DR, Abdalla DR, Borges AM, et al: Cytokine serum levels in patients with cervical intraepithelial neoplasia grade II-III treated with intralesional interferon- $\alpha 2 \mathrm{~b}$. Tumori 97 578-584, 2011.

7. Bednarek AK, Laflin KJ, Daniel RL, Liao Q, Hawkins KA and Aldaz CM: WWOX, a novel WW domain-containing protein mapping to human chromosome 16q23.3-24.1, a region frequently affected in breast cancer. Cancer Res 60: 2140-2145, 2000.

8. Del Mare S, Salah Z and Aqeilan RI: WWOX: its genomics, partners, and functions. J Cell Biochem 108: 737-745, 2009.

9. Aqeilan RI, Hagan JP, de Bruin A, et al: Targeted ablation of the WW domain-containing oxidoreductase tumor suppressor leads to impaired steroidogenesis. Endocrinology 150: 1530-1535, 2009.

10. Hsu LJ, Schultz L, Hong Q, et al: Transforming growth factor beta1 signaling via interaction with cell surface Hyal-2 and recruitment of WWOX/WOX1. J Biol Chem 284: 16049-16059, 2009.

11. Aqeilan RI, Pekarsky Y, Herrero JJ, et al: Functional association between Wwox tumor suppressor protein and p73, a p53 homolog. Proc Natl Acad Sci USA 101: 4401-4406, 2004.

12. Gaudio E, Palamarchuk A, Palumbo T, et al: Physical association with WWOX suppresses c-Jun transcriptional activity. Cancer Res 66: 11585-11589, 2006.

13. Li MY, Lai FJ, Hsu LJ, et al: Dramatic co-activation of WWOX/ WOX1 with CREB and NF-kappaB in delayed loss of small dorsal root ganglion neurons upon sciatic nerve transection in rats. PLoS One 4: e7820, 2009.

14. Guo W, Wang G, Dong Y, Guo Y, Kuang G and Dong Z: Decreased expression of WWOX in the development of esophageal squamous cell carcinoma. Mol Carcinog: Dec 27, 2011 (Epub ahead of print). doi: 10.1002/mc.21853.

15. Maeda N, Semba S, Nakayama S, Yanagihara K and Yokozaki H: Loss of WW domain-containing oxidoreductase expression in the progression and development of gastric carcinoma: clinical and histopathologic correlations. Virchows Arch 457: 423-432, 2010 .
16. Kuroki T, Yendamuri S, Trapasso F, et al: The tumor suppressor gene WWOX at FRA16D is involved in pancreatic carcinogenesis. Clin Cancer Res 10: 2459-2465, 2004.

17. Donati V, Fontanini G, Dell'Omodarme M, et al: WWOX expression in different histologic types and subtypes of non-small cell lung cancer. Clin Cancer Res 13: 884-891, 2007.

18. Giarnieri E, Zanesi N, Bottoni A, et al: Oncosuppressor proteins of fragile sites are reduced in cervical cancer. Cancer Lett 289: 40-45, 2010.

19. Guler G, Uner A, Guler N, et al: The fragile genes FHIT and WWOX are inactivated coordinately in invasive breast carcinoma. Cancer 100: 1605-1614, 2004.

20. Kurek KC, Del Mare S, Salah Z, et al: Frequent attenuation of the WWOX tumor suppressor in osteosarcoma is associated with increased tumorigenicity and aberrant RUNX2 expression. Cancer Res 70: 5577-5586, 2010.

21. Porter AG and Jänicke RU: Emerging roles of caspase-3 in apoptosis. Cell Death Differ 6: 99-104, 1999.

22. Kerr JF, Wyllie AH and Currie AR: Apoptosis: a basic biological phenomenon with wide-ranging implications in tissue kinetics. Br J Cancer 26: 239-257, 1972.

23. Evan G and Littlewood T: A matter of life and cell death. Science 281: 1317-1322, 1998.

24. Arnoult D, Gaume B, Karbowski M, Sharpe JC, Cecconi F and Youle RJ: Mitochondrial release of AIF and EndoG requires caspase activation downstream of Bax/Bak-mediated permeabilization. EMBO J 22: 4385-4399, 2003.

25. Liu X, Kim CN, Yang J, Jemmerson R and Wang X: Induction of apoptotic program in cell-free extracts: requirement for dATP and cytochrome c. Cell 86: 147-157, 1996.

26. Zou H, Henzel WJ, Liu X, Lutschg A and Wang X: Apaf-1, a human protein homologous to $C$. elegans CED-4, participates in cytochrome c-dependent activation of caspase-3. Cell 90: 405-413, 1997.

27. Hu BS, Tan JW, Zhu GH, Wang DF, Zhou X and Sun ZQ: WWOX induces apoptosis and inhibits proliferation of human hepatoma cell line SMMC-7721. World J Gastroenterol 18: 3020-3026, 2012.

28. Zhang P, Jia R, Ying L, et al: WWOX-mediated apoptosis in A549 cells mainly involves the mitochondrial pathway. Mol Med Rep 6: 121-124, 2012.

29. Chiang MF, Yeh ST, Liao HF, Chang NS and Chen YJ: Overexpression of WW domain-containing oxidoreductase WOX1 preferentially induces apoptosis in human glioblastoma cells harboring mutant p53. Biomed Pharmacother 66: 433-438, 2012.

30. Finnis M, Dayan S, Hobson L, et al: Common chromosomal fragile site FRA16D mutation in cancer cells. Hum Mol Genet 14: 1341-1349, 2005

31. Jee KJ, Kim YT, Kim KR, Aalto Y and Knuutila S: Amplification at $9 \mathrm{p}$ in cervical carcinoma by comparative genomic hybridization. Anal Cell Pathol 22: 159-163, 2001.

32. Tsuda H, Takarabe T, Okada S, et al: Different pattern of loss of heterozygosity among endocervical-type adenocarcinoma, endometrioid-type adenocarcinoma and adenoma malignum of the uterine cervix. Int J Cancer 98: 713-717, 2002.

33. Cullen AP, Reid R, Campion M and Lörincz AT: Analysis of the physical state of different human papillomavirus DNAs in intraepithelial and invasive cervical neoplasm. J Virol 65: 606-612, 1991.

34. Hopman AH, Smedts F, Dignef W, et al: Transition of high-grade cervical intraepithelial neoplasia to micro-invasive carcinoma is characterized by integration of HPV $16 / 18$ and numerical chromosome abnormalities. J Pathol 202: 23-33, 2004.

35. Crook T, Wrede D, Tidy JA, Mason WP, Evans DJ and Vousden KH: Clonal p53 mutation in primary cervical cancer: association with human-papillomavirus-negative tumours. Lancet 339: 1070-1073, 1992. 